\title{
Erratum
}

\section{Leaning in and speaking up? Students' perceptions of female leadership in healthcare}

[Physiotherapy Practice and Research 40 (2) (2019), pp. 167-176; DOI:10.3233/PPR-190138]

https://content.iospress.com/articles/physiotherapy-practice-and-research/ppr190138

Emer McGowan and Emma Stokes

Discipline of Physiotherapy, Trinity College, The University of Dublin, Ireland

When this article was originally published, a footnote incorrectly suggested that Dr Stokes carried out this research in her capacity as the President of the World Confederation for Physical Therapy. This information should have been included under the 'Other disclosure' section. 